\title{
On the dynamics and breakup of a bubble immersed in a turbulent flow
}

\author{
F. Ravelet, ${ }^{1,2, \text { a) }}$ C. Colin, ${ }^{1}$ and F. Risso ${ }^{1, b)}$ \\ ${ }^{1)}$ Institut de Mécanique des Fluides de Toulouse, Université de Toulouse and CNRS, Allée du Prof. C. Soula, \\ 31400 Toulouse, France. \\ 2) Arts et Metiers ParisTech, DynFluid, 151 boulevard de l'Hôpital, 75013 Paris, \\ France.
}

(Dated: Submitted: 1 July 2011)

Experimental investigations of the dynamics of a deformable bubble rising in a uniform turbulent flow are reported. The turbulence is characterized by fast PIV. Time-resolved evolutions of bubble translation, rotation and deformation are determined by three-dimensional shape recognition from three perpendicular camera views. The bubble dynamics involves three mechanisms fairly decoupled: (i) average shape is imposed by the mean motion of the bubble relative to liquid; (ii) wake instability generates almost periodic oscillations of velocity and orientation; (iii) turbulence causes random deformations that sometimes lead to breakup. The deformation dynamics is radically different from that observed in the absence of a significant sliding motion due to buoyancy. Large deformations that lead to breakup are not axisymmetric and correspond to elongations in the horizontal direction. The timescale of decay of shape oscillations is of the same order as their natural frequency $f_{2}$, so that breakup always results from the interaction with a single turbulent eddy. This overdamping causes the statistics of large deformations and the statistics of breakup identical to the statistics of turbulence. The bubble response time $f_{2}^{-1}$ however controls the duration of individual breakup events.

\section{INTRODUCTION}

In the absence of a strong rise velocity of the bubble due to buoyancy, the deformation of a bubble in a turbulent flow results from the response of surface modes to the turbulent forcing ${ }^{1,2}$. The deformation is well described by axisymmetric mode two ${ }^{3}$, the shape of which corresponds to the spherical harmonic of order 2 , the frequency and damping rate of which are well predicted by the linear theory ${ }^{4,5}$. The turbulent forcing is well described by the instantaneous turbulent Weber number experienced by the bubble along its trajectory. Furthermore, the breakup is observed to occur when the instantaneous deformation reaches a critical value. Due to the major role played by the bubble dynamics, the statistics of bubble shape and breakup occurrence are radically different from those of turbulence.

One open question is whether this description is still valid for a large bubble rising in water. Even in the absence of turbulence, a large rising bubble is no more spherical but flattened in the vertical direction. This may change the natural frequency and the damping rate of the oscillations ${ }^{6-8}$. Moreover, due to the instability of its wake, the bubble does not rise on a straight path but follows helical or zigzagging path ${ }^{9}$. Shape oscillations also occur and are observed to involve non-axisymmetric $\operatorname{modes}^{10,11}$.

When a rising bubble is immersed in a turbulent flow, the action of the turbulent fluctuations is thus combined to both a non-zero average deformation and selfsustained oscillations.

\footnotetext{
a) Electronic mail: florent.ravelet@ensta.org

b) Electronic mail: frederic.risso@.imft.fr
}

This paper reports an experimental investigation on the dynamics of a rising bubble in a turbulent flow. The objective is to determine how a bubble moves and deforms under the combined action of its rise velocity, wakeinduced oscillations and turbulent fluctuations. First, this requires an experimental set-up in which a rising bubble can be observed over a long time. Following the idea of Wichterle et al. ${ }^{12}$, this has been obtained by immersing a bubble in a slightly rotating downward flow through a slightly divergent pipe. Second, it also requires to generate a calibrated turbulent flow with an intensity large enough to cause the bubble breakup. Ideally, this flow should be as uniform as possible to avoid considering changes in turbulence properties as the bubble moves. Third, we need to track the three-dimensional motion and shape of the bubbles, which requires timeresolved records of at least three perpendicular view of the bubble.

The experimental setup is presented in $\S$ II. An original technique of image processing has been developed to track the three-dimensional dynamics and is presented in $\S$ III. The turbulent flow has been characterized with fast Particle Image Velocimetry and the results are presented in $\S \mathrm{IV}$. The section $\mathrm{V}$ is devoted to the study of bubbles dynamics at moderate deformations. The breakup process is finally analyzed in $\S \mathrm{VI}$ and concluding remarks are given in $\S \mathrm{VII}$.

\section{EXPERIMENTAL SETUP}

The experimental device consists of a closed flow loop. A sketch is shown in Fig. 1. The test section is a vertical conical pipe out of Plexiglas in which water flows downward. It is of height $320 \mathrm{~mm}$ with an inner diameter of $50 \mathrm{~mm}$ at inlet, and $80 \mathrm{~mm}$ at outlet (half-angle of the 
cone is thus $2.7^{\circ}$ ). Perturbations are generated in the flow with a rotating grid, made with four blades (right trapezoid of height $45 \mathrm{~mm}$, top side $40 \mathrm{~mm}$ and bottom side $22 \mathrm{~mm}$ ). This impeller is surrounded by a converging conical casing, leaving a layer of $2 \mathrm{~mm}$ between the blade tips and the wall. The bottom of this conical housing is the $z$ reference (see Fig. 1). The grid consists of $3 \mathrm{~mm}$ holes every $5 \mathrm{~mm}$ in an hexagonal pattern, and thus has a solidity (ratio of blocked area and unit area) of 0.67 . The grid is rotated between 1 and $3 \mathrm{~Hz}$, corresponding to tip speeds in the range $0.25-0.75 \mathrm{~m} \cdot \mathrm{s}^{-1}$.
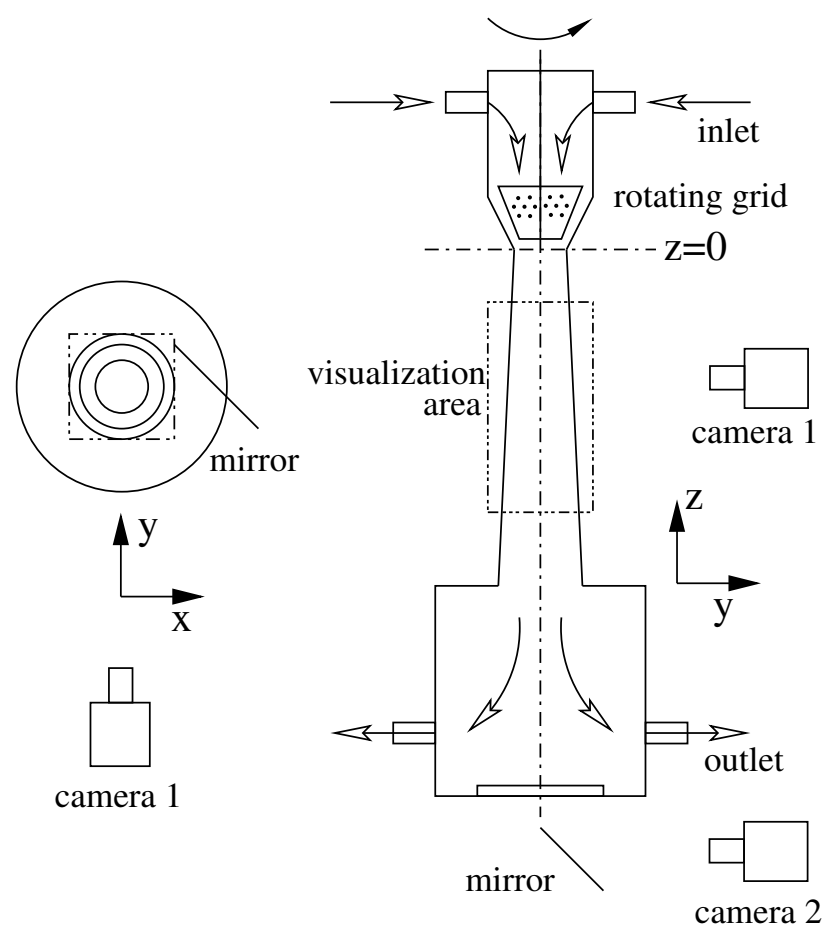

FIG. 1. Sketch of the experimental setup. Left: top view. Camera 1 gives two side views in the planes $x-z$ and $y-z$ with the help of a $45^{\circ}$ mirror. The visualization area is surrounded by a square glass box filled with water. Right: side view. Camera 2 gives the bottom view in a plane $x-y$ with the help of another $45^{\circ}$ mirror. Two LED plates of $120 \times 120 \mathrm{~mm}$ are used for lighting of the vertical planes, and the horizontal plane is illuminated with the help of an halogen lamp above the experiment. The rotating grid is covered with luminescent paint and is illuminated from its sides in order to have as bright as possible a background for the horizontal plane.

The flow rate, produced with a centrifugal pump is measured with a Bürker flow transmitter, and can be set in the range $0.5-0.7 \mathrm{~L} \cdot \mathrm{s}^{-1}$, corresponding to average velocities in the range $0.10-0.35 \mathrm{~m} \cdot \mathrm{s}^{-1}$ in the test section.

Air bubbles are injected at the bottom of the test section, with a pipe of inner diameter $4 \mathrm{~mm}$, at the end of which different pipe elbows can be connected. A way to get regular sized bubbles is to inject a known quantity of air with a motorized syringe and to have the exit of the elbow pointing down. In the study reported here, the bubbles have an equivalent diameter of $d=9.3 \pm 0.3 \mathrm{~mm}$.

The visualization volume extends from $z=-100 \mathrm{~mm}$ to $z=-180 \mathrm{~mm}$ and is surrounded by a square glass box filled with water to minimize optical distortions. Two cameras and two mirrors are used to get three different views of the bubble in the visualization volume (see Fig. 1). Two components of the flow field have been measured in several planes with fast Particle Image Velocimetry (PIV). More details about the PIV measurements are given in $\S \mathrm{IV}$.

\section{IMAGE PROCESSING}

Every experiment with bubbles consists of a sequence of 2000 images taken simultaneously by the two cameras, at a rate of $300 \mathrm{~Hz}$. In order to extract quantitative information about the bubble shape and its dynamics, the three images taken by the two cameras are processed under Matlab software following the procedure illustrated in Figs. 2 and 3. This procedure can be decomposed into three steps: ellipses that are equivalent to the side views of the bubble on camera 1 are first defined using simple algorithm, then an ellipse equivalent to the bottom view on camera 2 is fitted with a different algorithm, and at last a resulting ellipsoid is rebuilt from these three perpendicular projection contours. Quantities such as the velocity of the center of mass or the large-scale deformation, that is relevant in the context of breakup mechanism, are then computed from the equivalent ellipsoid.

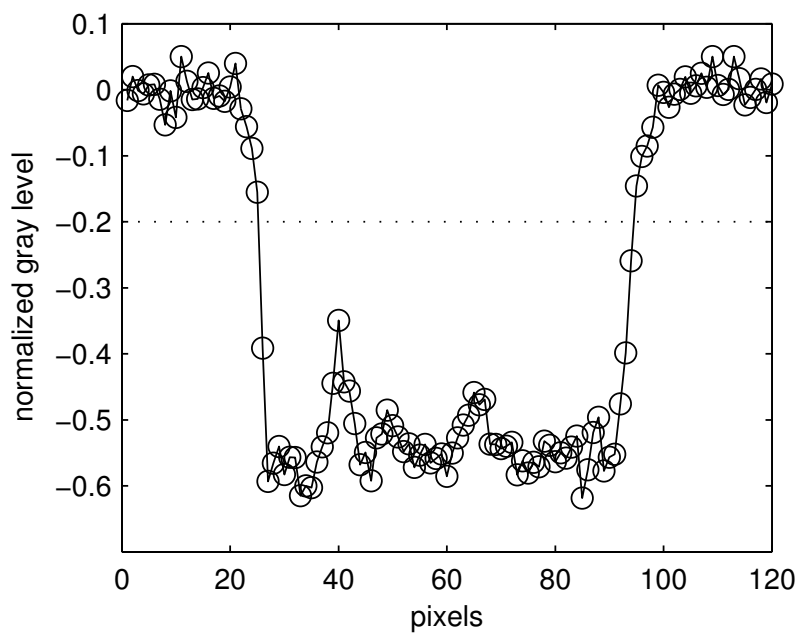

FIG. 2. Normalized gray level profile along constant height $z=-146.5 \mathrm{~mm}$ for view 1 of image 39 of sequence $B 5$ (see also top left quadrant in Fig. 3). Horizontal dotted line: threshold used for binarization. Bubble corresponds to values below the threshold. Calibration factor: 6.5 pixels corresponds to $1 \mathrm{~mm}$. 

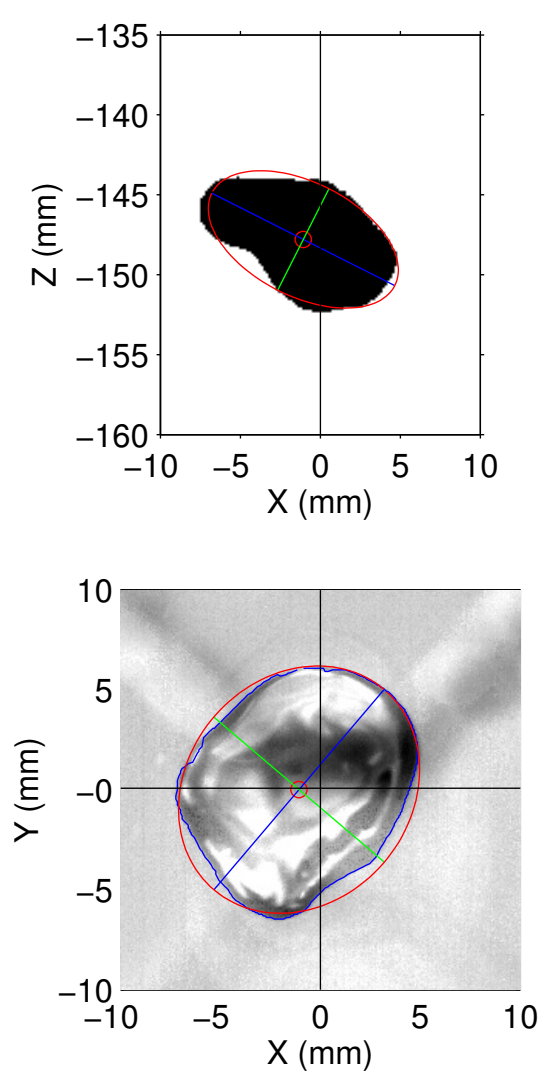
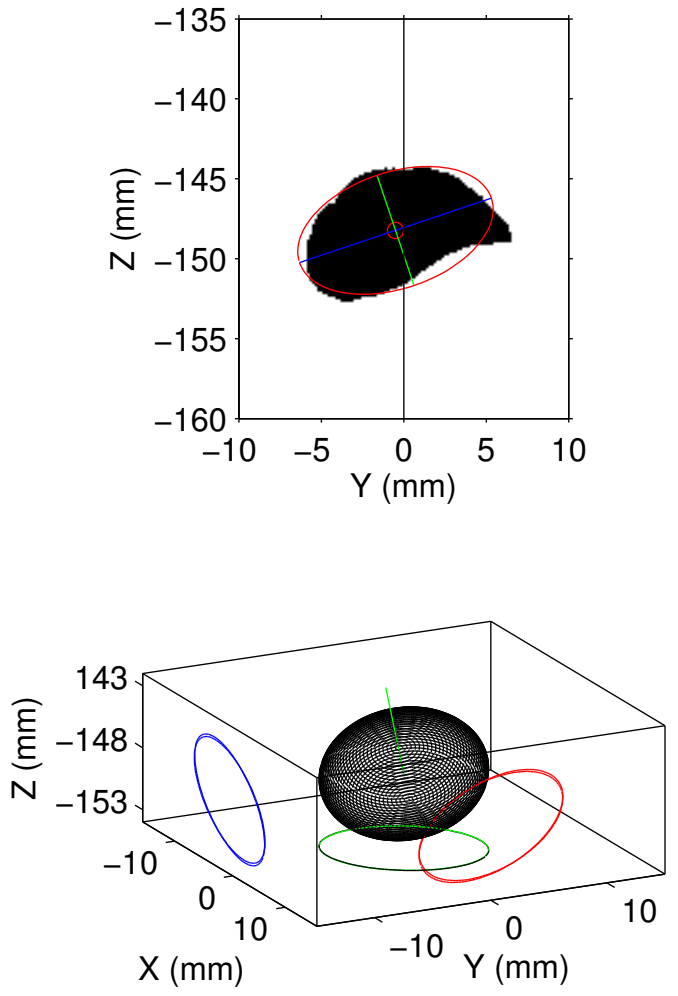

FIG. 3. Illustration of image processing on image 39 of sequence $B 5$ (see Tab. I). Top left: side view in the plane $x-z$ (camera 1) with the equivalent ellipse (red line), its center of mass (red dot) and major (blue line) and minor axes (green line). Top right: side view in the plane $y-z$ (camera 1) with the same conventions. Bottom left: bottom view of camera 2 in the plane $x-y$ with the Snake active contour (blue line) and the ellipse fitted on this contour (red line) with its center of mass (red dot) and major (blue line) and minor axes (green line). Bottom right: ellipsoid reconstructed with the three projections. The ellipses used to compute the ellipsoid are plotted as solid lines on the three planes together with the three projections of the reconstructed ellipsoid, which are displayed as dashed lines. Solid green line gives the direction of the ellipsoid minor axis.

\section{A. Algorithm for side views detection}

For the two side views of camera 1, we use simple binarization after subtraction and normalization by a background image taken without bubbles. The stability of the LED plates illumination is very good, and a single threshold of -0.20 has been used for all the images. An example showing the sharpness of the gray level profile at the bubble boundary can be found in Fig. 2. The typical number of pixels inside the detected bubble is around 3000. The center of mass and the second-order moments of the shape are then computed. That defines an equivalent ellipse displayed with red lines in the upper quadrants of Fig. 3. We have compared this method with some edge detection algorithms, to test the accuracy of the measured features. For the Canny method implemented in Matlab, the mean discrepancy between the two techniques is less than $1 \%$ for the area and for the length of the equivalent ellipse axes. The discrepancy is moreover less than $0.3^{\circ}$ for the orientation of the axes. The simple binarization has been retained because it is more robust and the threshold can be chosen once and for all.

\section{B. Algorithm for bottom view detection}

For the bottom view of camera 2, the problem is slightly different because of the lighting from the top with an halogen lamp and because of the presence of the rotating grid in the field of view. These constraints lead to poor contrast and render simple binarization with a single threshold impossible. The images of camera 2 are thus treated with the following procedure.

We first take a set of pictures without bubbles, and have thus a moving background which we synchronize and then subtract from the images, as shown in the lower left quadrant of Fig. 3. One can still guess the position of the rotating grid which is blurred in the background of the resulting image. The background is non-uniform and the contrast between the bubble and the background is not very high. However one can distinguish the con- 
tour of the bubble, finding sharp edges and rebuilding the contour by extrapolation.

We have implemented such a method with Matlab. The first step uses a SuSan edge detection algorithm ${ }^{13}$ that has been implemented in $\mathrm{C}$ for efficiency. The values for the kernel radius (3 pixels) and for the threshold $(10 \%)$ that are used are claimed to be optimal and universal $^{13}$. Only sharp edges are captured by this algorithm and there are still holes in the contour. We then use active contours to capture the bubble interface. We use a greedy snake algorithm ${ }^{14}$, with weighting parameters $\alpha=0.3$ for continuity/elasticity, $\beta=0.7$ for curvature/rigidity and $\gamma=3$ for the attraction to edge map. The snake is initialized by hand for the first image of a sequence, and then dilated in every direction by an amount of 10 pixels from the converged state to give an initial value for the next image. These parameters were modified by hand for images close to breakup where the projection of the bubble on the horizontal plane has strong concave curvatures. An ellipse is then fitted on the converged snake contour (see blue and red lines in the lower left quadrant of Fig. 3).

\section{Ellipsoid reconstruction and relevant parameters}

The ellipses that have thus been fitted on the projections of the bubble in three perpendicular planes give 9 parameters. An ellipsoid has 6 independent parameters (3 lengths and 3 orientations). It is thus possible to reconstruct an equivalent ellipsoid by minimization ${ }^{15}$. The lower right quadrant of Fig. 3 presents the reconstructed ellipsoid, the three ellipses used to determine it, and the three back projections of the ellipsoid onto the planes. One can notice that they hardly distinguish from each other. We have defined an error function for the reconstructed shape as the maximum of the radial distance between the fitted ellipses and the back-projected ellipses. The point is rejected when the error exceeds $0.8 \mathrm{~mm}$, i.e. roughly $10 \%$ of the semi major axis length.

The whole process requires ten hours of computation on a desktop computer for the first pass on a sequence of 2000 images. After this first pass, typically $5 \%$ of the images have been rejected and are retreated by hand in a second pass. The final rate of rejection excluding the breakup phase is below $1 \%$ for all the sequences.

The physical quantities that are extracted from the image processing may help to understand how the turbulence affects the deformation of the bubble. The rising bubbles that are studied are relatively large and are thus no more spherical. Their wake is moreover unstable and this leads to a complicated path ${ }^{9}$ that may also affect the deformation dynamics. The extracted quantities are thus the lengths of the three semi-axes of the ellipsoid $(a, b$ and $c)$, the velocity of the center of mass $V$ that is computed as the derivative of the position, and the orientation $\theta$ of the short axis of the ellipsoid with respect to the vertical axis.

\section{CHARACTERIZATION OF THE FLOW FIELD}

\section{A. Mean features of the large-scale flow}

The flow field has been measured in the absence of bubbles with 2D-2C fast PIV in one vertical plane containing the symmetry axis, then in eight horizontal planes, $10 \mathrm{~mm}$ apart from each other. The data acquisition rate is $600 \mathrm{~Hz}$ and time series of 6000 images have been recorded. The PIV analysis consists of standard correlation of two successive images on windows of $32 \times 32$ pixels with an overlap of $50 \%$ in a first pass, and then on windows of $16 \times 16$ pixels with $0 \%$ overlap in two successive passes. Normalized median test ${ }^{16}$ has been used to discard outliers, which were reconstructed with a bilinear interpolation. The rate of outliers is always below $4 \%$.

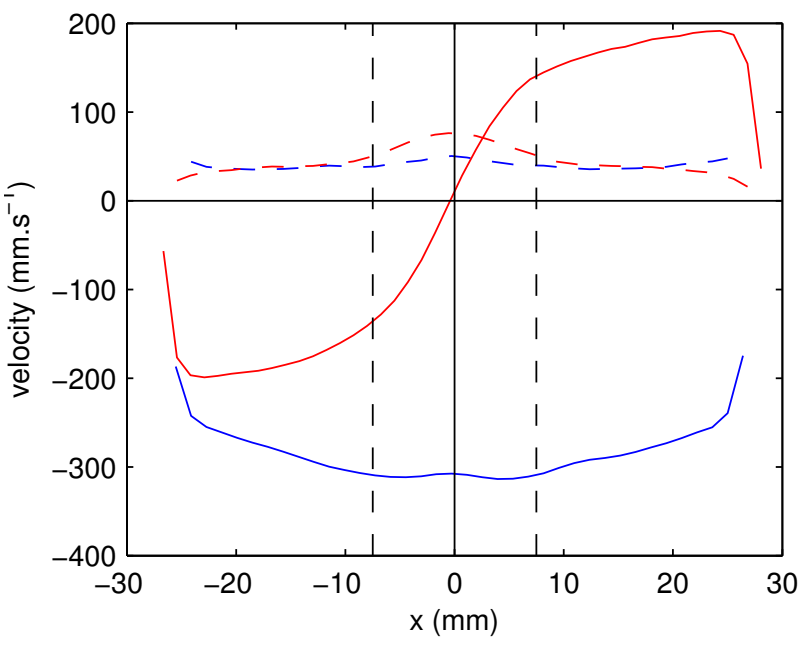

FIG. 4. Velocity profiles at $z=-140 \mathrm{~mm}$ along $x$-axis, for a flow rate of $0.62 \mathrm{~L} \cdot \mathrm{s}^{-1}$ and a rotation rate of $1.5 \mathrm{~Hz}$. Blue: vertical velocity $u_{z}$ (solid line) and its standard deviation $\sigma_{u_{z}}$ (dashed line). Red: tangential velocity $u_{y}$ (solid line) and its standard deviation $\sigma_{u_{y}}$ (dashed line). The vertical dashed lines stand for the core region of diameter $15 \mathrm{~mm}$.

The mean flow consists of an axial spreading jet superimposed with rotation. We have studied three cases: a case with rotating grid and no mean flow rate, a second case with an imposed mean flow rate and no rotation, and the case with both pumping and rotation which is relevant for bubble dynamics and breakup studies. In the experiments reported here, the flow rate is $0.62 \mathrm{~L} \cdot \mathrm{s}^{-1}$ and if so, the rotation rate of the rotating grid is $1.5 \mathrm{~Hz}$.

The first two cases are first briefly discussed. The flow is turbulent in every case. On the one hand, when the grid does not rotate the mean vertical particle displacement is of the order of 6 pixels - or $-300 \mathrm{~mm} \cdot \mathrm{s}^{-1}$ — in the middle of the test section, and the mean flow is axisymmetric. The standard deviation of the vertical component of the velocity $\sigma_{u_{z}}$ is around $8 \%$ of the time-averaged velocity everywhere, while a typical PIV evaluation error 


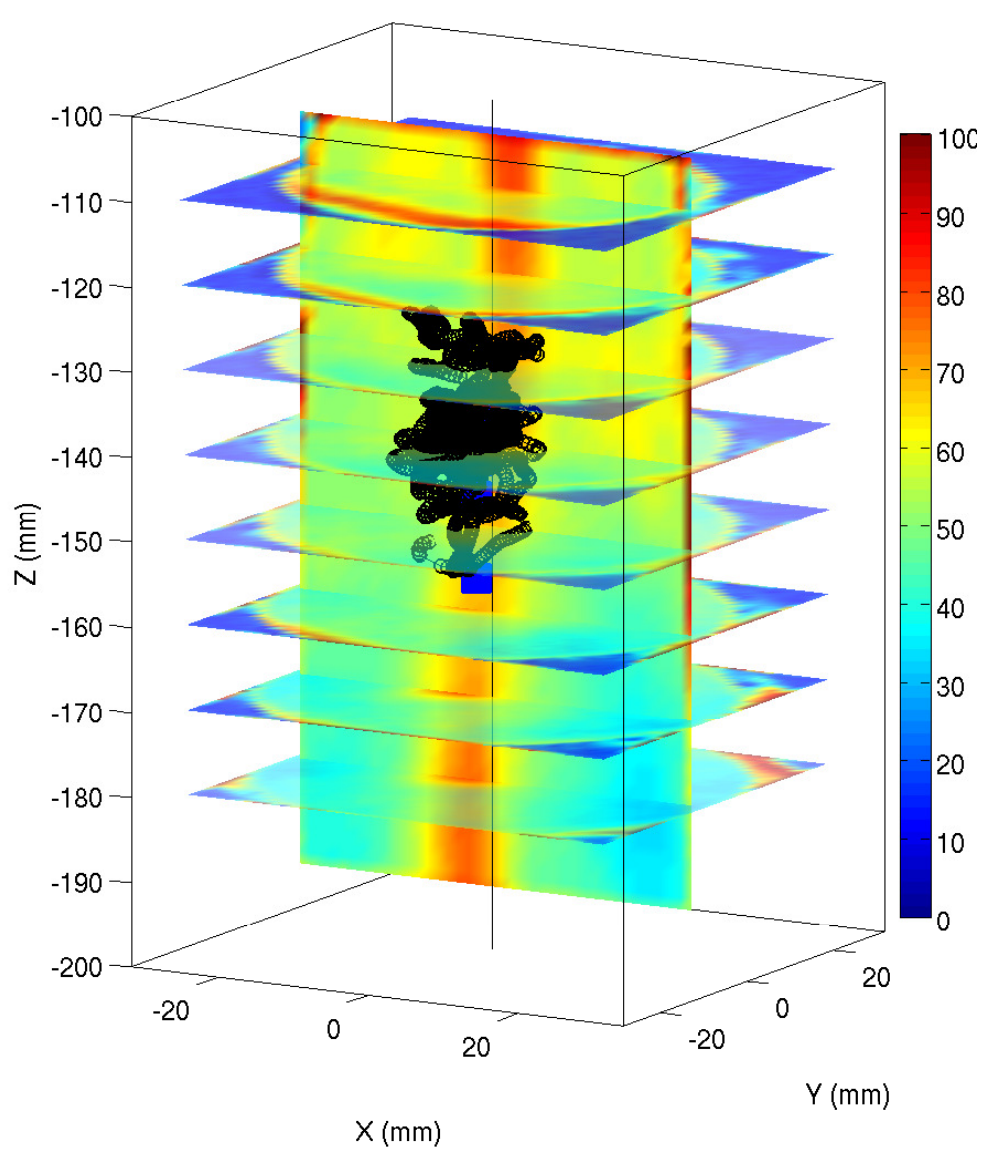

FIG. 5. Standard deviation of the velocity measured in one vertical plane and eight horizontal planes superimposed with the trajectory of bubble $B 5$ (black) and mean positions of the six bubbles (blue squares).

of 0.1 pixel corresponds to a fluctuation rate of $2 \%$. On the other hand, when the grid rotates alone it creates an upward pumping of typical velocity $45 \mathrm{~mm} \cdot \mathrm{s}^{-1}$ on the center line with recirculation cells close to the cone edges. The flow is moreover no more axisymmetric. And last, the flow in the center line is very fluctuating (fluctuation rate $35 \%$ ).

The Figures 4, 5 and 6 present velocity measurements that have been performed in the relevant case with pumping and rotation. The vertical and horizontal velocity profiles along the $x$-axis at $z=-140 \mathrm{~mm}$ are plotted in Fig. 4. The mean axial jet is directed downward and has a flat profile of constant velocity around $u_{z} \simeq-300 \mathrm{~mm} \cdot \mathrm{s}^{-1}$. The fluctuation rate of the vertical component of the velocity is above $10 \%$ and even exceeds $20 \%$ in the core region.

Concerning the horizontal velocities, the grid creates solid body rotation within an annulus of diameter $15 \mathrm{~mm}$. The horizontal velocities are of the order of $2 / 3$ of the vertical velocity, and in the center, their fluctuation rates are of the order of $35 \%$ of the maximal horizontal speed, i.e. of the order of $20 \%$ of the vertical speed. The mean flow is not axisymmetric as can be seen in Fig. 5 where the center-line of the vortex slightly deviates from the $z$-axis. The bubbles are however trapped inside the vortex annular core in a zone that extends from $z \simeq-110 \mathrm{~mm}$ to $z \simeq-160 \mathrm{~mm}$ (see Fig. 5). The rotation rate inside the vortex core that is deduced from velocity measurements is bigger than the impeller rotation rate of $1.5 \mathrm{~Hz}$ : the velocity measured $20 \mathrm{~mm}$ away from the center corresponds to $2 \mathrm{~Hz}$ at $z=-100 \mathrm{~mm}$, and to $1.6 \mathrm{~Hz}$ at $z=-180 \mathrm{~mm}$. This can be due to the fact that the impeller is a trapezoid tightly enclosed in a housing.

A time signal of the vertical velocity at a point close to the center of the region where the bubbles are trapped is plotted in Fig. 6 together with the corresponding power spectrum. The flow is turbulent and the spectrum exhibits a large inertial range with a $-5 / 3$ power law. The integral time scale $\Theta$ that could be estimated from the autocorrelation function of the time signal in Fig. 6 is $\Theta \simeq 0.10$ s, i.e. an integral length scale $\Lambda \simeq 30 \mathrm{~mm}$ deduced from the Taylor's hypothesis of frozen turbulence. This approximately corresponds to three times the bubble diameter, which is close to $10 \mathrm{~mm}$. The estimated 

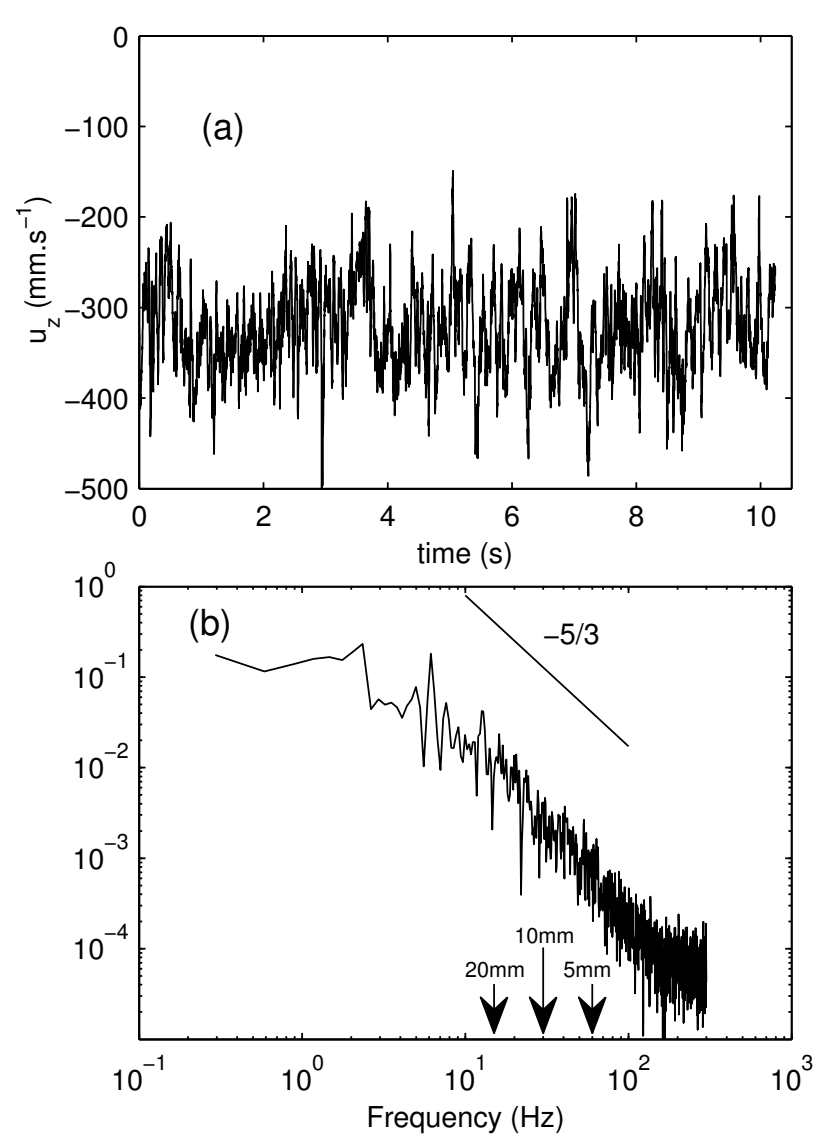

FIG. 6. Velocity measurements for a flow rate of $0.62 \mathrm{~L} \cdot \mathrm{s}^{-1}$ and a rotation rate of $1.5 \mathrm{~Hz}$. (a): time signal of the vertical velocity $u_{z}$ at point $x=-0.3, y=0, z=-140$ (in $\mathrm{mm}$ ). (b): corresponding power spectrum. Arrows stand for corresponding length scales using Taylor's hypothesis.

Taylor micro-scale is roughly $0.016 \mathrm{~s}$, that corresponds to $\lambda \simeq 5 \mathrm{~mm}$, i.e. half the bubble diameter. The arrows in Fig. 6b that stand for three length scales surrounding the mean bubble diameter are in the inertial range of the spectrum. The bubble thus locally experiments the turbulent nature of the flow.

In conclusion, the bubbles are trapped within a core region of $15 \mathrm{~mm}$ width where they experience a turbulent flow that is roughly isotropic: the fluctuations of velocity are of the same order of magnitude $\left(60 \mathrm{~mm} \cdot \mathrm{s}^{-1}\right)$ for the three components of the velocity. The mean axial flow is moreover homogeneous in this region, and the horizontal velocity resembles solid-body rotation.

\section{B. Consequences on the bubbles}

With these velocity measurements, we can now estimate the ratio of deforming forces and stabilizing forces acting on the bubbles. For our air bubbles in water, the viscous shear is negligible: a capillary number $\mathrm{Ca}$ based on the measured shear rate of the mean flow, on the viscosity of water and on the interfacial tension between air and water $\sigma=70 \mathrm{mN} \cdot \mathrm{m}^{-1}$ is $C a \leq 5 \times 10^{-4}$. The relevant numbers are thus Weber numbers comparing inertia to capillarity.

The bubble of mean diameter $d=9.3 \pm 0.3 \mathrm{~mm}$ has a mean rising velocity $U_{b}=300 \mathrm{~mm} \cdot \mathrm{s}^{-1}$ and is in a region where the fluid is in solid body rotation at angular velocity $\Omega=9.4 \mathrm{rad} \cdot \mathrm{s}^{-1}$. The bubble Reynolds number is $R e_{b}=U_{b} d / \nu \simeq 2800$ with $\nu=10^{-6} \mathrm{~m}^{2} \cdot \mathrm{s}^{-1}$ the kinematic viscosity of water. The Weber number based on the mean rising velocity is $W e=\rho d U^{2} / \sigma \simeq 11.6$, with $\rho=10^{3} \mathrm{~kg} \cdot \mathrm{m}^{-3}$ the density of the continuous phase. These values of $R e_{b}$ and $W e$ correspond to a situation with a large mean deformation of the bubble that should be an elongated ellipsoid rising with an oscillating path ${ }^{8,9}$ as confirmed by the results discussed in $\S \mathrm{V}$. The Weber number $W e_{\Omega}$ based on the solid body rotation is very small: $W e_{\Omega}=\rho d(d \Omega / 2)^{2} / \sigma \simeq 0.25$. The solid body rotation plays no role in the deformation of the bubble. It will actually maintain the bubble in the central zone of the flow (see Fig. 5).

The eigenfrequency of the mode 2 for the deforma-

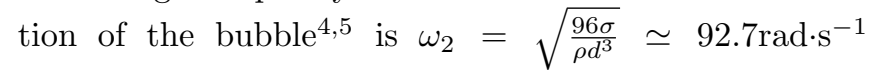
$\left(f_{2}=14.8 \mathrm{~Hz}\right)$. In the following, all the temporal features will be compared to this time scale. The Reynolds number based on this oscillation frequency is $R e_{o s c}=$ $1 / 2 d^{2} \omega_{2} / \nu \simeq 3900$. It is fairly high, which implies a small damping rate of the free oscillations of a bubble at rest $^{4,5}: \beta=\frac{80 \nu}{d^{2}} \simeq 0.9 \mathrm{~s}^{-1}$. The rising velocity of the bubble is thus of the same order of magnitude as the shape oscillation velocity: $R e_{b} / R e_{o s c}=0.7$. The rising velocity may thus have significant effects on the shape dynamics. Finally, the level of turbulent fluctuations in the velocity field is high $\left(\sigma_{u} / U_{b} \simeq 1 / 5\right)$ and may also have a significant impact on the shape dynamics.

To take into account the contribution of turbulent fluctuations, we define an instantaneous turbulent Weber number $W e_{t}(\mathbf{x}, t)$ at each point, based on the velocity scale $\delta \hat{u}^{2}(\mathbf{x}, t)=\max \left(\mathrm{u}^{\prime}(\mathbf{x}+\mathbf{b}, \mathrm{t})-\mathrm{u}^{\prime}(\mathbf{x}-\mathbf{b}, \mathrm{t})\right)^{2}$. This velocity scale $\delta \hat{u}$ is the maximum of the second order structures functions computed for distance $d$ (b is a vector of length $d / 2$ and various orientations, the maximum being taken on these orientations). The spatial distribution of this turbulent Weber number is very similar to the distribution of the velocity fluctuations that is presented in Fig. 5, and the time-averaged value of the turbulent Weber number is less than 1.8 in the test region.

The temporal features of this signal at a point close to the center of the visualization volume are given in Fig. 7. At this point, the mean turbulent Weber number is 1.3 and the standard deviation is 1.2 . One can notice in Fig. 7b that the probability density function of $W e_{t}$ is skewed towards high values: very rare events with high values of the turbulent Weber number arise. One event is highlighted in the inset of Fig. 7a: we notice that the Weber number exceeds a significant value $\left(W e_{t} \geq 5\right)$ 
for a non negligible time with respect to the time scale given by $f_{2}$, that is roughly $W e_{t} \geq 5$ during $0.75 f_{2}{ }^{-1}$. The distribution of the waiting times between these high $W e_{t}$ events resembles a Poisson process as can be seen in Fig. 7c where the distribution of time intervals between two successive events with $W e_{t} \geq 2$ is reported. The distribution is exponential with a time constant of $0.04 \mathrm{~s}$ or $0.6 f_{2}^{-1}$ in this case.
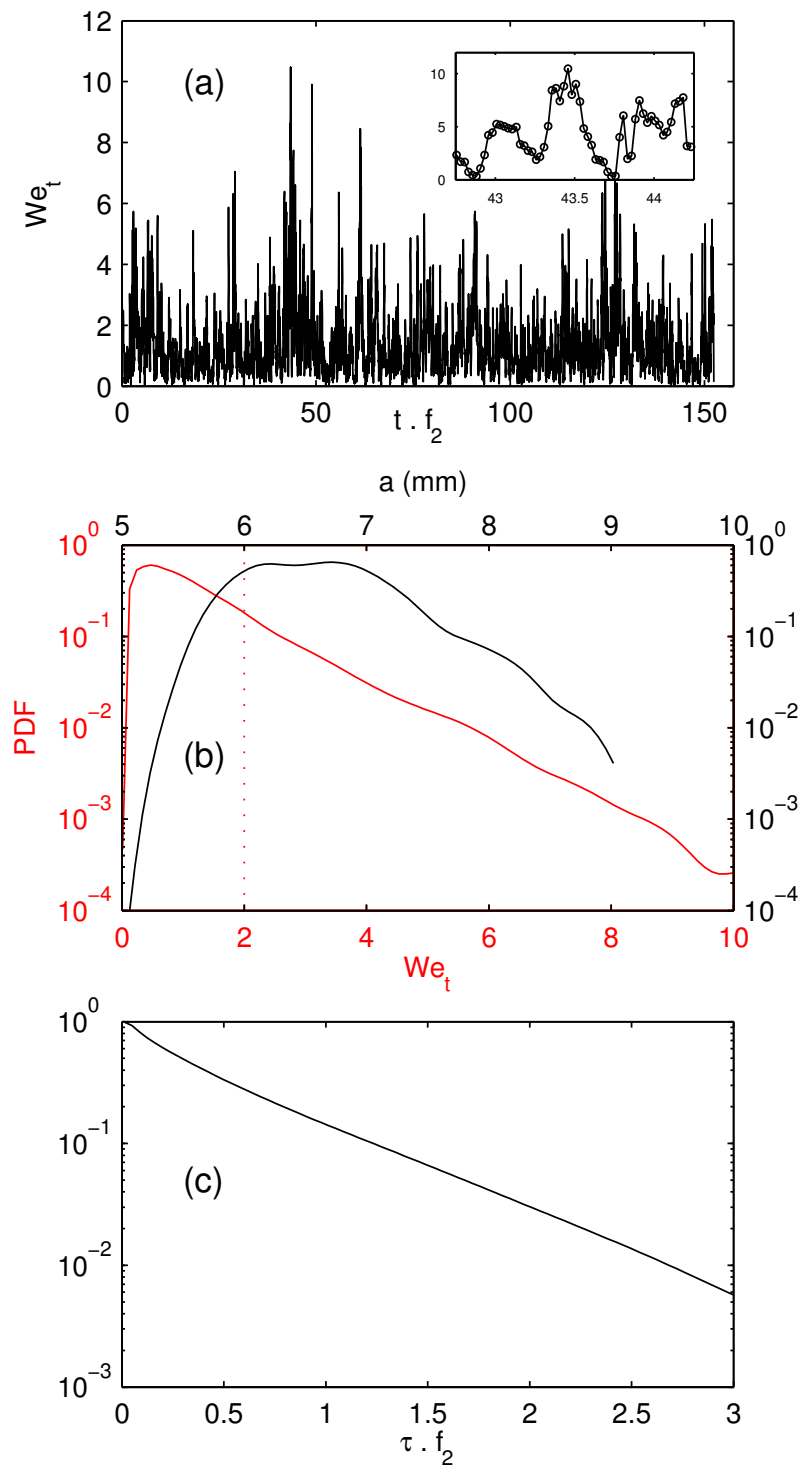

FIG. 7. Measurement of the turbulent Weber number $W e_{t}(\mathbf{x}, t)$ at point $x=-0.3, y=0, z=-140$ (in $\mathrm{mm}$ ) for a flow rate of $0.62 \mathrm{~L} \cdot \mathrm{s}^{-1}$ and a rotation rate of $1.5 \mathrm{~Hz}$. (a): time signal. (b): corresponding probability density function, in red (left and bottom axis). The black curve (top and right axis) is the probability density function of major axis $a$ for bubble B5. (c): distribution of time intervals between two successive events corresponding to $W e_{t} \geq 2$. This threshold is highlighted by the vertical dotted line in (b). All the times are made non-dimensional with $f_{2} \simeq 15 \mathrm{~Hz}$.

\section{Introduction to the study of bubble dynamics}

The mean features of the large-scale flow and the turbulent statistics that may be relevant towards the shape dynamics of bubbles of mean diameter $9.3 \mathrm{~mm}$ have thus been characterized. The following two sections deal with the bubble shape dynamics. As described thereafter, the breakup rate is very low and the breakup process does not seem to depend on the history of the bubble - in other words no resonant breakup is observed. The dynamics during moderate deformations phases is thus first presented $(\oint \mathrm{V})$, and the breakup phase is then specifically analyzed $(\S \mathrm{VI})$.

\section{DYNAMICS OF MODERATE DEFORMATIONS}

This section is devoted to the behavior of bubbles of typical diameter $d=9.3 \pm 0.3 \mathrm{~mm}$ that are injected in the previously described velocity field, excluding the breakup phase. The results reported hereafter concern six sequences of 2000 images taken at $300 \mathrm{~Hz}$ for six independent bubbles which dimensions are presented in Tab. I. These values have been extracted from the images with the procedure described in $\S$ III. A sequence of 25 side view images in the plane $x-z$ (camera 1$)$ is displayed in Fig. 8 and may be considered as a typical example to illustrate the deformations and motion of the bubble. This sequence has been chosen because the major axis of the equivalent ellipsoid lies in this $x-z$ plane during the selected time interval. The time series of the three ellipsoid semi-axis, of the horizontal velocity $V_{x}$ and of the orientation of the short axis $\theta$ for sequence $B 5$ are moreover plotted in Fig. 9.

\begin{tabular}{cccccccccc}
\hline \hline Bubble & $\langle a\rangle$ & $\langle b\rangle$ & $\langle c\rangle$ & $\left\langle(a b c)^{1 / 3}\right\rangle$ & $\chi$ & $\sigma_{a}$ & $\sigma_{b}$ & $\sigma_{c}$ & $\sigma_{a b c^{1 / 3}}$ \\
\hline$B 1$ & 6.05 & 4.98 & 3.05 & 4.49 & 1.83 & 0.48 & 0.38 & 0.29 & 0.07 \\
$B 2$ & 6.10 & 4.94 & 3.04 & 4.49 & 1.84 & 0.52 & 0.40 & 0.30 & 0.09 \\
$B 3$ & 6.22 & 5.13 & 3.14 & 4.62 & 1.84 & 0.52 & 0.41 & 0.33 & 0.09 \\
$B 4$ & 6.50 & 5.24 & 3.11 & 4.71 & 1.92 & 0.67 & 0.45 & 0.34 & 0.12 \\
$B 5$ & 6.65 & 5.39 & 3.19 & 4.82 & 1.92 & 0.62 & 0.49 & 0.35 & 0.13 \\
$B 6$ & 7.03 & 5.49 & 3.00 & 4.84 & 2.14 & 0.75 & 0.55 & 0.38 & 0.13 \\
\hline \hline
\end{tabular}

TABLE I. Fitted ellipsoids dimensions in mm for six bubbles. Mean half major axis $\langle a\rangle$, half medium axis $\langle b\rangle$, half short axis $\langle c\rangle$. Equivalent radius $\left\langle(a b c)^{1 / 3}\right\rangle$. Aspect ratio $\chi=\langle(a+$ $b) / 2 c\rangle$. Standard deviations $\sigma_{a}, \sigma_{b}, \sigma_{c}$ and $\sigma_{a b c^{1 / 3}}$.

\section{Mean shape}

The instantaneous shape of the bubble strongly differs from an ellipsoid as can be seen in Fig. 8. The semiaxis lengths are moreover strongly fluctuating (Fig. 9c): the fluctuating rate of the three axes are of the order of $10 \%$. One can also notice in Tab. I that the volume of 

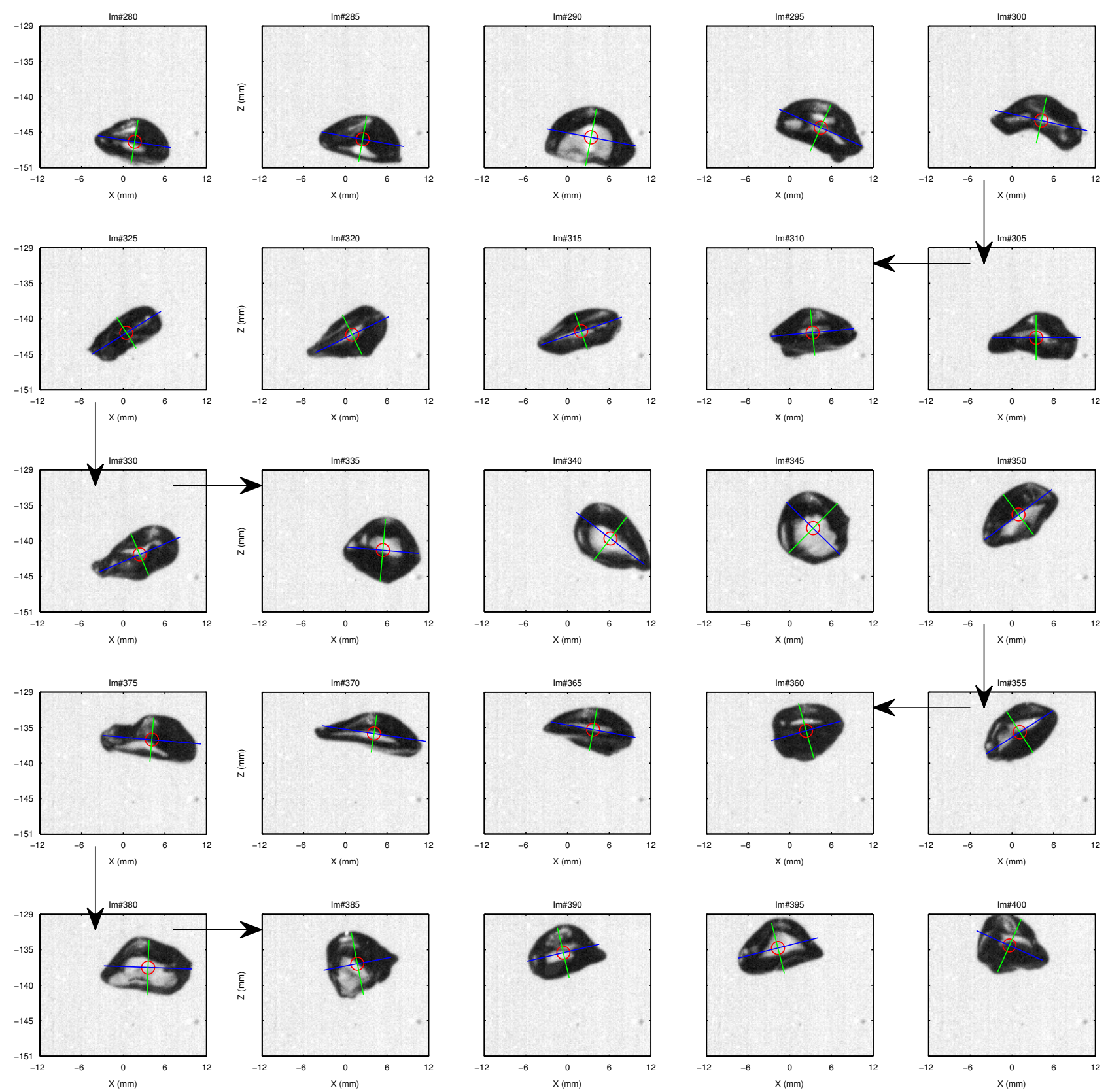

FIG. 8. Illustration of a deformation sequence on case $B 5$. Side view in the plane $x-z$ (camera 1 ) with the center of mass (red dot), the major (blue line) and minor axes (green line) of the equivalent ellipse. One image over 5 is displayed and the time lag between each image thus corresponds to $1 / 60$ s $\left(\left(4 f_{2}\right)^{-1}\right)$. Please note that the sequence reads from top left to top right then the second line reads from right to left, and so on, following the arrows. 

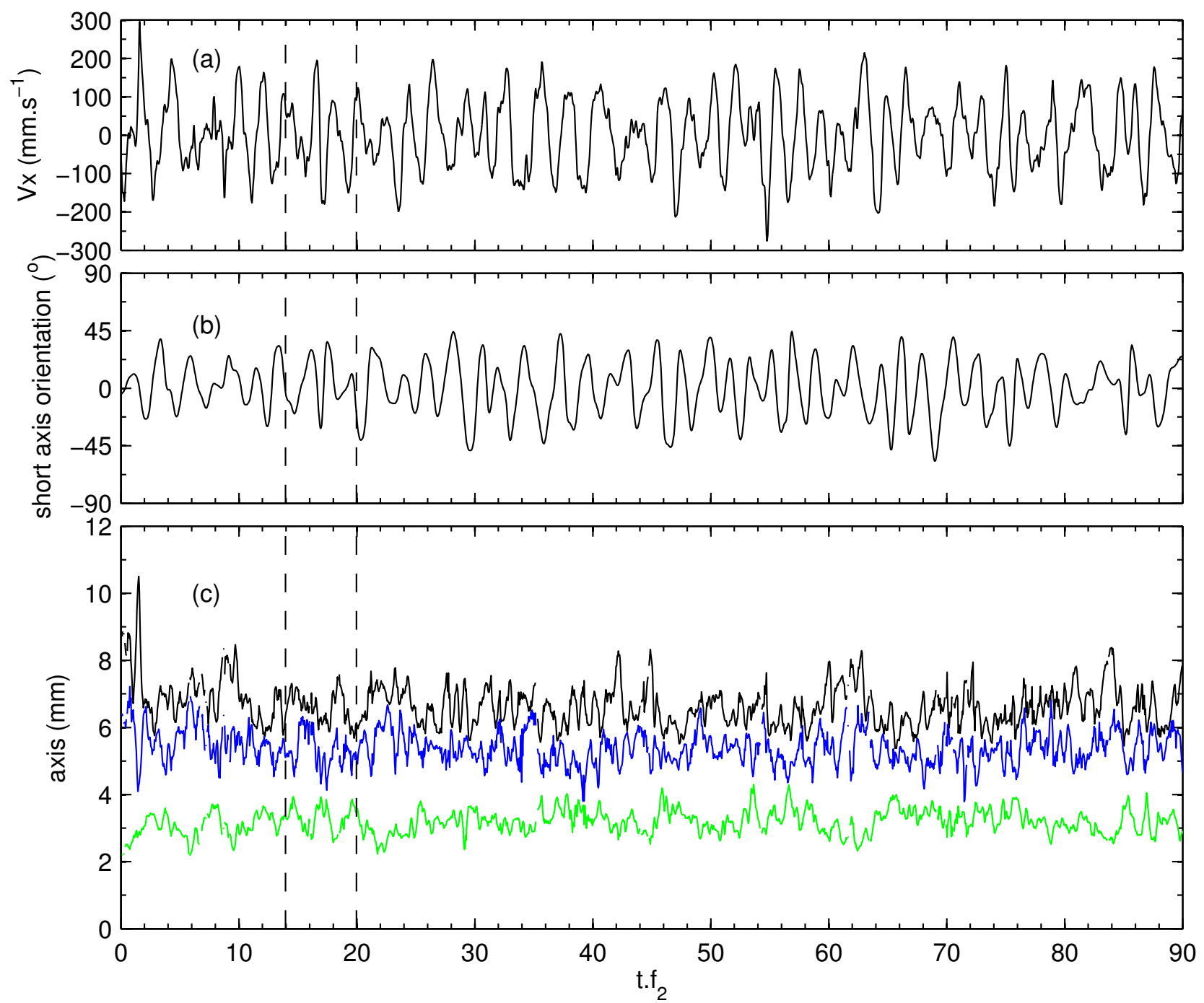

FIG. 9. Time signals of (a): horizontal velocity $V_{x}$, (b): orientation $\theta$ of the short axis relative to vertical direction, and (c): semi-axis lengths (black: $a$, blue: $b$, green: $c$ ) for sequence $B 5$. All the times are made non-dimensional with $f_{2} \simeq 15 \mathrm{~Hz}$. The vertical dashed lines stand for the time interval corresponding to the sequence depicted in Fig. 8.

the equivalent ellipsoid is not conserved: the fluctuation rate of $(a b c)^{1 / 3}$ is nevertheless small, around $2 \%$. Finally, the following observations can be drawn:

- the mean shape of the bubbles is an ellipsoid with a symmetry axis around the short axis;

- the mean aspect ratio of this ellipsoid $\chi$ is $1.91 \pm$ 0.11 and seems to increase with the equivalent radius;

- the short axis is pointing in the vertical direction on average, as can be seen in Fig. 9b.

\section{Position and motion of the bubble}

The mean positions of the six bubbles are displayed with blue squares in Fig. 5 . The trajectory of bubble $B 5$ is also diplayed in this figure. The trajectory of a bubble is quite complicated and frequently exhibits loops but the six bubbles are however trapped inside the vortex annular core as described in $\S$ IV. The fluctuations of the position are of the same order of magnitude for every bubble: the standard deviation of the vertical position is $\sigma_{z} \simeq 7 \mathrm{~mm}$ and the fluctuations in the horizontal positions are $\sigma_{x}=$ $\sigma_{y} \simeq 4 \mathrm{~mm}$. These last two could be translated in polar coordinates centered on the mean bubble position and would give a fluctuation of radial position $\sigma_{r} \simeq 2.5 \mathrm{~mm}$.

Though the bubble path seems erratic, the time series of the horizontal velocity (Fig. 9a) and of the short axis 
orientation (Fig. 9b) are more regular and may exhibit a periodic behavior. The horizontal velocity has a standard deviation of the order of $100 \mathrm{~mm} \cdot \mathrm{s}^{-1}$. The temporal spectra of horizontal velocity and of orientation are plotted in Fig. 10. Some peaks are clearly visible and give a typical harmonic component for the path at $8.5 \mathrm{~Hz}$. The crosscorrelation between $V_{x}$ and $\theta$ moreover shows a peak at -0.73 , with a time delay between two successive peaks in the cross-correlation function that corresponds to this frequency of $8.5 \mathrm{~Hz}$. This behavior is consistent with a wake instability. The strouhal number built with this frequency, the mean bubble rising velocity and the bubble diameter is $S t=\frac{f d}{U_{b}} \simeq 0.27$. According to the works of Lindt ${ }^{17}$, the prediction for the strouhal number of the wake is $S t=0.28$.

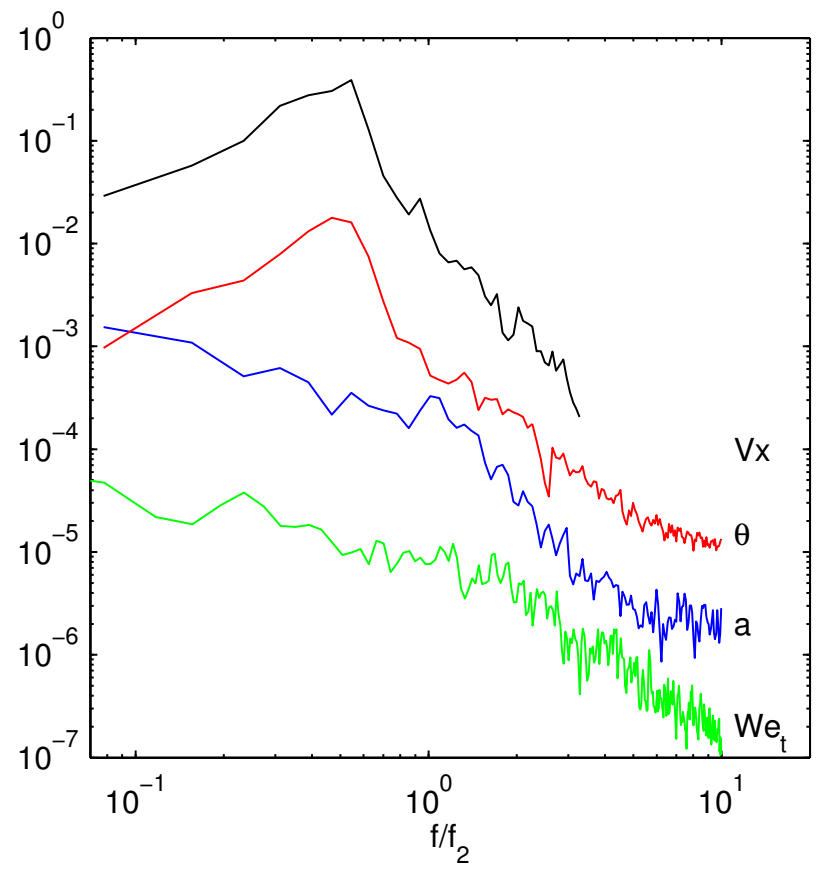

FIG. 10. Power spectra of (black): $V_{x}$, (red): $\theta$, (blue): $a$ for sequence $B 5$ (please see also the time signals in Fig. 9) and (green): $W e_{t}$ at point $x=-0.3, y=0, z=-140 \mathrm{~mm}$ (please see the time signal in Fig. 7). The frequencies are made nondimensional with $f_{2} \simeq 15 \mathrm{~Hz}$. The different spectra have been vertically shifted for the sake of clarity.

\section{Dynamics of deformation}

The significant deformations that the bubbles undergo on the contrary do not exhibit any periodic behavior, as can be seen in Fig. 9c on the time series of the three semi-axes of the equivalent ellipsoid and in Fig. 10 where no peak is visible in the power spectrum of $a$. There thus seem to be no trace of a periodic shape oscillation at the eigenfrequency $f_{2}$ at first glance, contrary to what is observed without buoyancy ${ }^{1,2}$. The deformations still have a part of deterministic dynamics that is highlighted with the analysis of the cross-correlations between the three axes lengths.

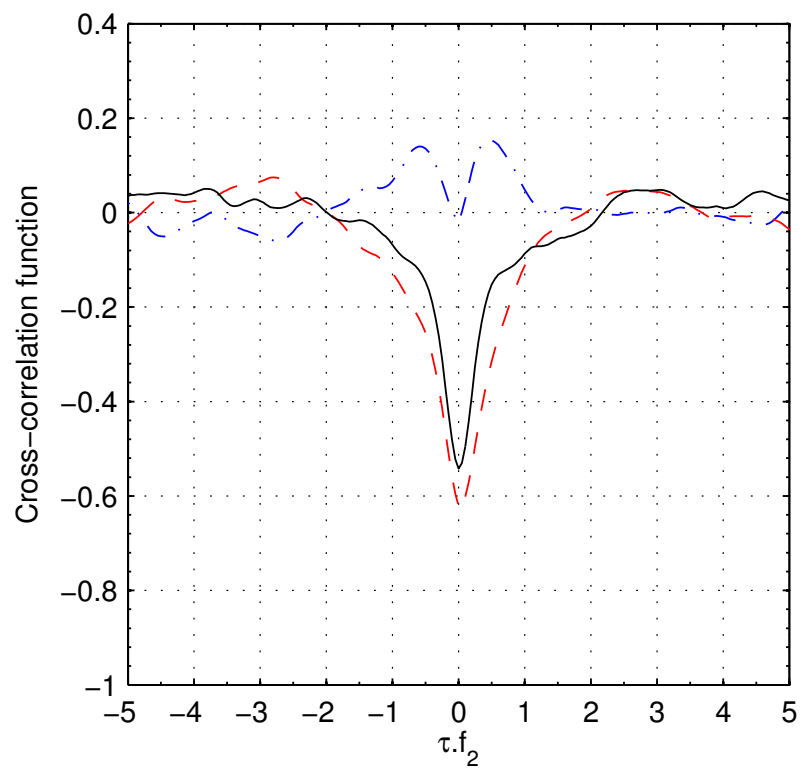

FIG. 11. Cross-correlation functions of (blue dash-dotted): $a-b$, (red dashed): $a-c$, and (black): $b-c$. An average has been made on all the sequences excluding breakup phases. The time lag is made non-dimensional with $f_{2} \simeq 15 \mathrm{~Hz}$.

The Figure 11 presents an average on all sequences excluding breakup phases of the cross-correlation functions of the two largest axes lengths $a-b$ (blue dash-dotted line), of the big and short axes lengths $a-c$ (red dashed line) and of the medium and short axes lengths $b-c$ (black line). The cross-correlation function is defined as:

$$
C_{a-b}(\tau)=\frac{\langle a(t) b(t-\tau)\rangle}{\sigma_{a} \sigma_{b}}
$$

where \langle\rangle stands for time average, $\tau$ is a time lag, and $\sigma$ is the standard deviation. The short axis is significantly anti-correlated to the two biggest axes, at 0 time-lag. The cross-correlation coefficient of $a$ and $c$, and $b$ and $c$ is indeed close to -0.6 at $\tau=0\left(C_{a-c}(\tau=0) \simeq-0.62\right.$ and $\left.C_{b-c}(\tau=0) \simeq-0.54\right)$. Concerning the two biggest axes, they have very few correlation: their cross-correlation coefficient at $\tau=0$ is $C_{a-b}(\tau=0) \simeq 0$. The deformations of the bubbles are thus non-axisymmetric, as already observed for large rising bubbles ${ }^{10,11}$. The cross-correlation function $C_{a-b}$ presents two local maxima around 0.15 for $\tau \simeq-0.6$ and $\tau \simeq 0.5$. This may be a trace of exchanges between $a$ and $b$ when the two main axes are close in length. Finally, one can notice that the cross-correlation function goes to zero rapidly with increasing time lags: one can estimate that the dynamics of the three axes is uncorrelated after 1 to $2 f_{2}^{-1}$. 
The short axis thus seems to play a particular role with respect to the deformations. As previously noticed, it is pointing in the vertical direction on average, i.e. it is aligned with the gravity. The analysis of the correlations suggest that the significant deformations take place in the horizontal direction and are not axi-symmetric, and that though no oscillations are observed, the characteristic time based on the eigenfrequency $f_{2}$ should be present.

In order to check this hypothesis, the following section will focus on the dynamics of the most intense deformations and on the breakup process.

\section{LARGE DEFORMATIONS AND BREAKUP}

The probability distribution function (PDF) of $a$ for bubble $B 5$ is plotted in black in Fig. 7b. This PDF is clearly not Gaussian and is asymmetric: the PDF has a positive skewness 0.5. A number of events that correspond to high values of $a$ should thus be detectable. We propose $a \geq\langle a\rangle+1.5 \sigma_{a}$, i.e. $a \geq 7.5 \mathrm{~mm}$ for bubble $B 5$, as an arbitrary threshold to qualify a large deformation. One question is to what extent these events are rare or

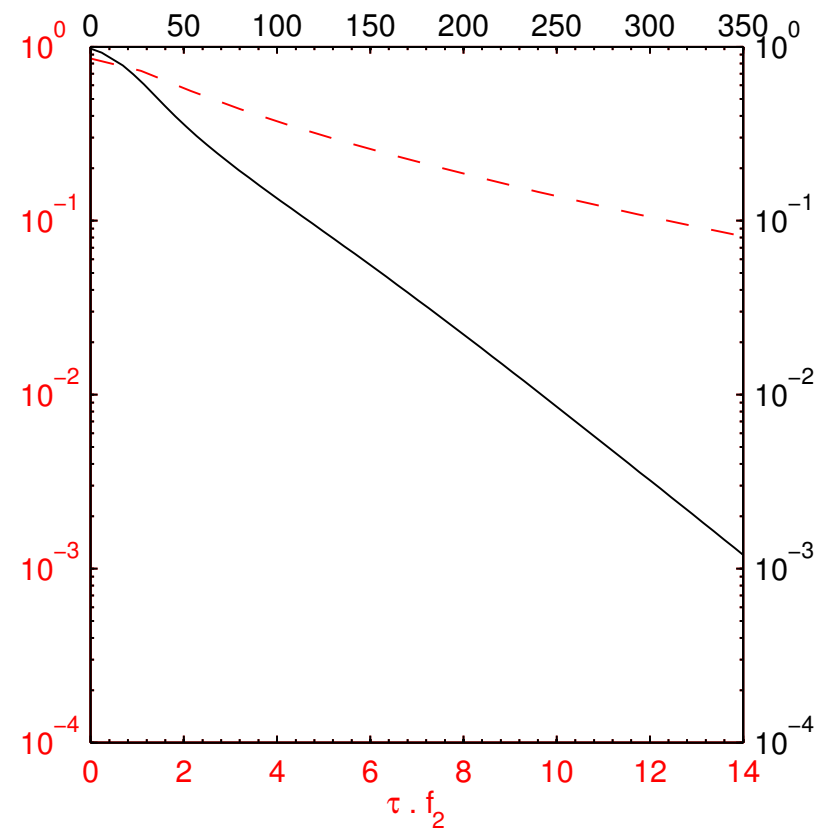

FIG. 12. Red dashed line, bottom and left axis: distribution of time intervals between two successive overtakings of a threshold for $a(a \geq 7.5 \mathrm{~mm})$, for sequence $B 5$. Black solid line, top and right axis: distribution of bubbles breakup times, for 76 breakup time measurements. (Please note that for breakup statistics, the velocity field is the same but the equivalent diameter of the bubbles has been increased to $12 \mathrm{~mm}$, in order to increase breakup rate. In that case, the time-scale that has been used to present dimensionless times is based on $f_{2} \simeq 10 \mathrm{~Hz}$ ).
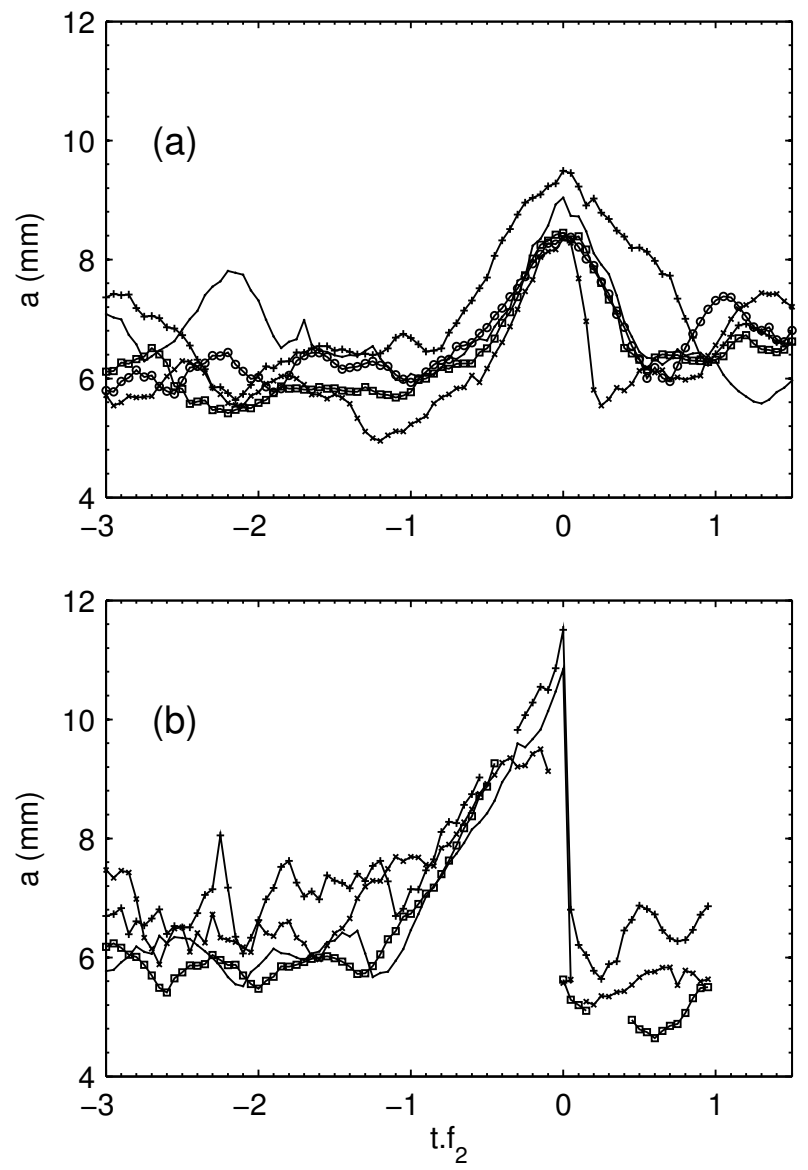

FIG. 13. Superposition of large deformation phases (a) and breakup events (b) for the different bubbles. The sequences have been synchronized on the maximum of $a$. The time is made non-dimensional with $f_{2} \simeq 15 \mathrm{~Hz}$. (a): case $B 2$ ( $\square$ and $\circ)$, case $B 3(\times)$, case $B 4$ (black line) and case $B 6(+)$. (b): case $B 2(\square)$, case $B 3(\times)$, case $B 4$ (black line) and case $B 6$ $(+)$.

frequent.

The distribution of the waiting times between two overtakings of this threshold is plotted in red in Fig. 12. The statistics is exponential with a characteristic time around $7 f_{2}^{-1}$. This behavior is reminiscent of the statistics for the turbulent Weber number that have been shown in Fig. 7c. For bubbles of equivalent diameter $9.3 \mathrm{~mm}$, the breakup rate is however very low, of the order of $50 \mathrm{~s}^{-1}$. In order to allow the determination of breakup statistics in a reasonable time, experimental measurements for the same flow conditions, but with larger bubbles, have been carried out. The black curve in Fig. 12 shows the statistics of breakup time of bubbles of equivalent diameter $12 \mathrm{~mm}$. These measurements show that the distribution of the life-time before breakup is also an exponential law. This is consistent with waiting for an instantaneous turbulent Weber number bigger than a certain threshold to lead to breakup. 

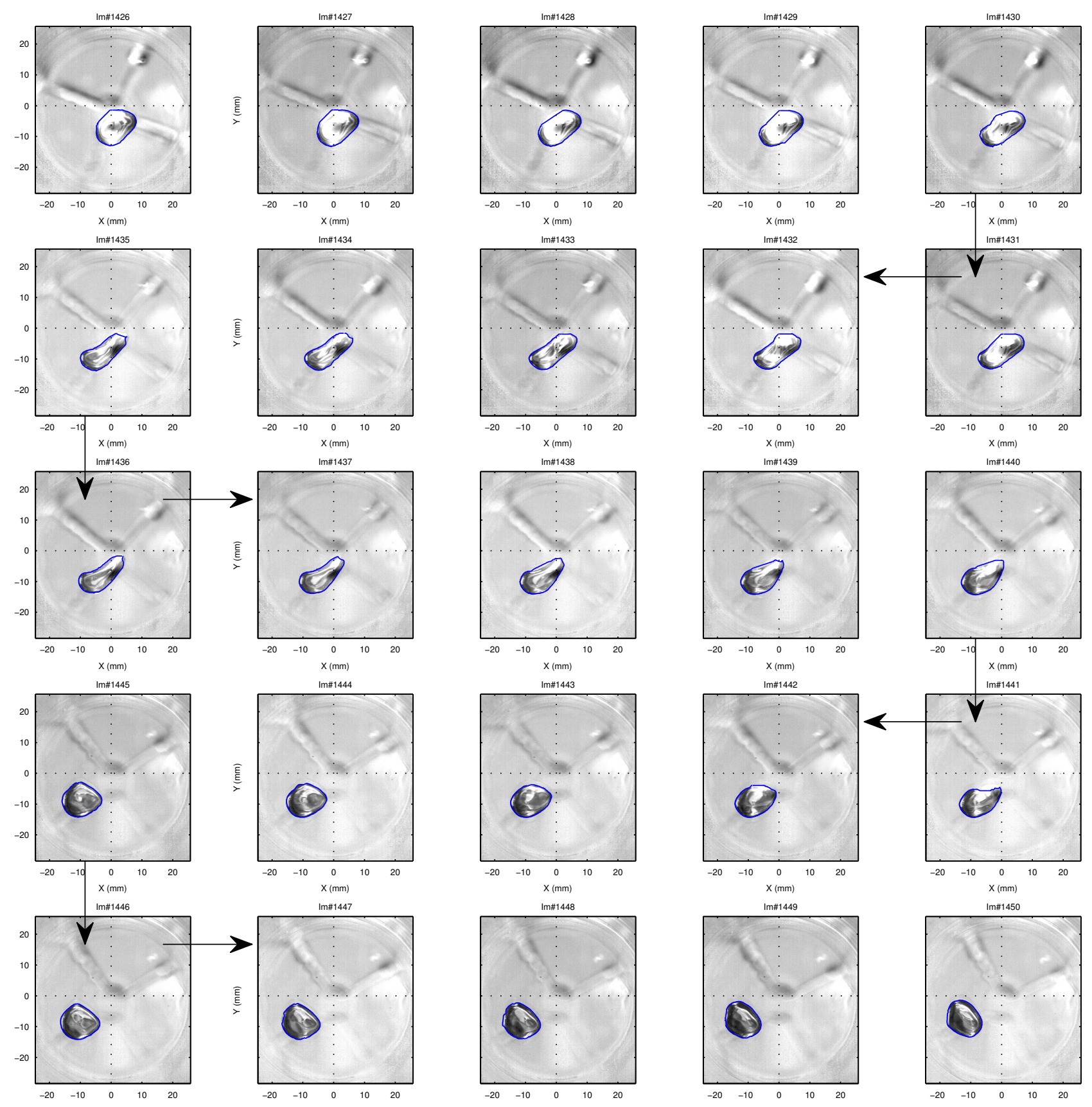

FIG. 14. Illustration of a large deformation sequence that do not lead to breakup on case $B 4$. Bottom view in the plane $x-y$ (camera 2) with the detected contour in blue. The time lag between each image corresponds to $1 / 300 \mathrm{~s}\left(\left(20 f_{2}\right)^{-1}\right)$. Please note that the sequence reads from top left to top right then the second line reads from right to left, and so on, following the arrows. The maximum deformation is reached in picture \#1434 with $a \simeq 9 \mathrm{~mm}$ and thus corresponds to time $t_{0}$ in Fig. 13 . 

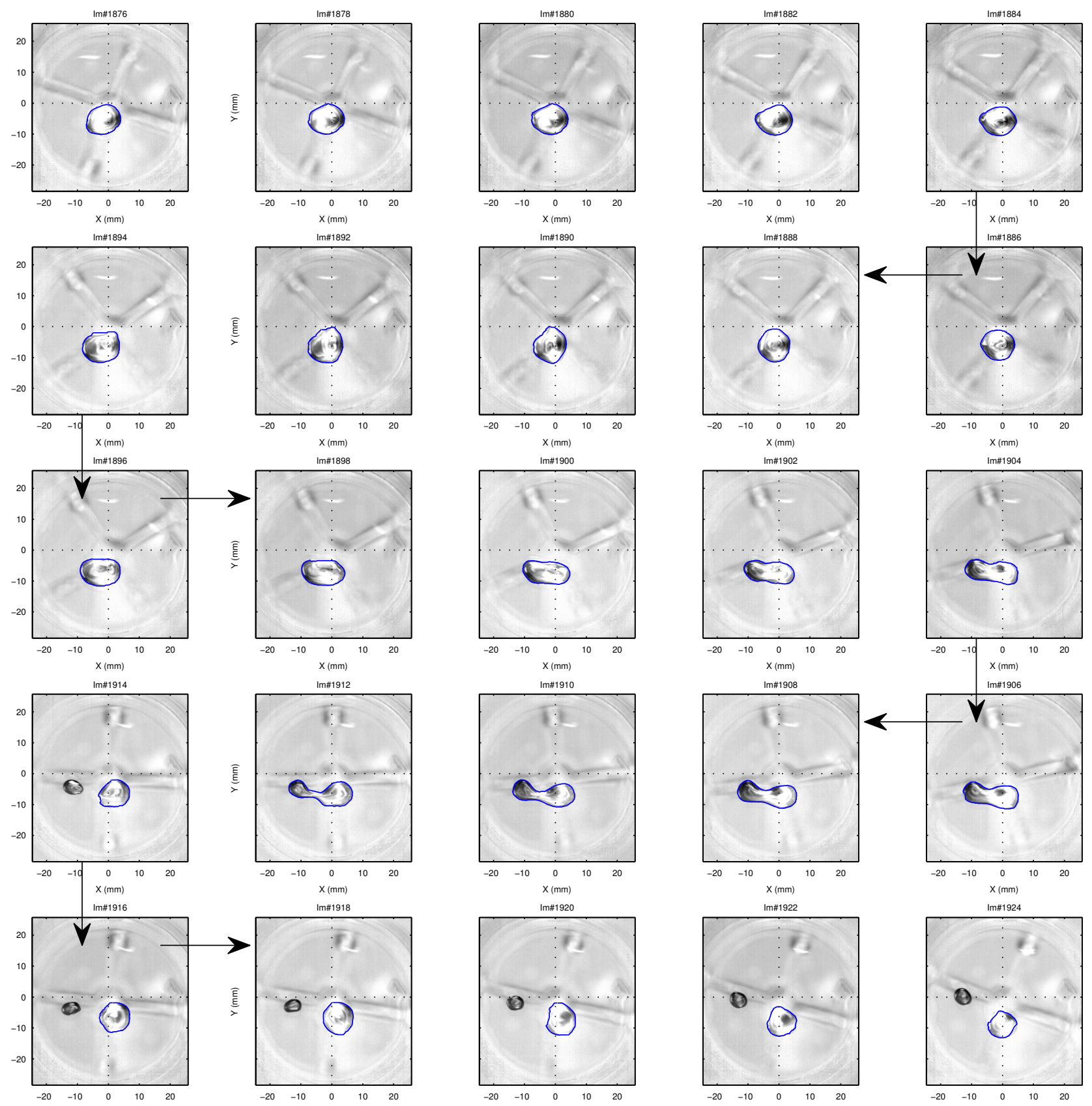

FIG. 15. Illustration of a breakup on case B2. Bottom view in the plane $x-y$ (camera 2) with the detected contour in blue. One image over 2 is displayed and the time lag between each image thus corresponds to $1 / 150$ s $\left(\left(10 f_{2}\right)^{-1}\right)$. Please note that the sequence reads from top left to top right then the second line reads from right to left, and so on, following the arrows. 
Some large deformations that do not lead to breakup have been identified and selected for the six experiments. They correspond to maximum deformations $8.3 \lesssim a \lesssim$ $9.5 \mathrm{~mm}$. The time series of $a$ have been synchronized to a reference time $t_{0}$ that corresponds to the maximum of $a$ and are gathered in Fig. 13a. They are plotted between $t_{0}-3 f_{2}$ and $t_{0}+1 f_{2}$. The corresponding serie of images in the plane $x-y$ (camera 2 ) for the sequence concerning bubble $B 4$ is displayed in Fig. 14 .

These series show the following common features for the large deformations. Firstly there are neither successive amplifications of the deformation nor periodic shape oscillations at the eigenfrequency $f_{2}$ as observed when stochastic resonance occurs ${ }^{1}$. A strong increase of $a$ is immediately followed by a decrease back to the mean level. Once synchronized to the maximum deformation, the phases of growth and decay nevertheless exhibit a distinct time-scale around $f_{2}^{-1}$. This is reminiscent of the behavior of a strongly damped oscillator. The large deformations moreover correspond to strongly elongated shapes of the bubble as can be seen in Fig. 14. In that case, the three dimensional aspect ratio at maximum deformation in picture \#1434 is $\chi \simeq 2.5$, and the aspect ratio in the horizontal plane is $a / b \simeq 2.0$. For all the large deformations, the bubble is elongated in an almost horizontal direction, the short axis being vertical within $\pm 15^{\circ}$. This shape is not axisymmetric and its decomposition into spherical harmonics involves a major contribution of mode $n=2, m=2$ where $n$ stands for the poloidal wave number and $m$ stands for the azimuthal wave number.

Finally, the breakup phases have been identified and the time series of axis a synchronized to the breakup time are gathered in Fig. 13b. The corresponding serie of images in the plane $x-y$ (camera 2) for bubble $B 2$ is displayed in Fig. 15. The breakups are very similar to the previously described large deformations. The four bubbles break in a horizontal plane when $a$ exceeds the value $a_{\text {break }} \simeq 10.5 \mathrm{~mm}$, which corresponds to approximately twice the equivalent bubble radius. This value of the critical deformation leading to breakup is in agreement with results obtained in the absence of buoyancy for both a bubble in a homogeneous turbulence ${ }^{1}$ or a drop in a strongly heterogeneous turbulence. ${ }^{2}$

The final phase of growth of $a$ are surprisingly collapsing for the four cases, with a characteristic time of rising again on the order of $f_{2}^{-1}$.

\section{CONCLUDING REMARKS}

The purpose of this study was to determine the dynamics of a deformable bubble rising in a turbulent flow. In particular, we are interested in understanding the role of the sliding motion induced by buoyancy upon the bubble deformation and breakup.

In the absence of significant sliding motion, the shape dynamics of a bubble in a turbulent low-viscosity liquid was already fairly well understood ${ }^{1,2}$. The bubble essentially responds to turbulent eddies of scales comparable to its diameter by experiencing deformations described by the axisymmetric spherical harmonics $n=2, m=0$, where $n$ stands for the poloidal wave number and $m$ stands for the azimuthal wave number of the decomposition in spherical modes. After interaction with an intense turbulent eddy, either the bubble breaks or performs weakly-damped oscillation at the frequency $f_{2}$ given by the linear theory ${ }^{4,5}$ of shape oscillations for this mode. The shape dynamics can be modeled by a linear oscillator forced by turbulent fluctuations, which amplifies frequencies close to $f_{2}$ and filters out others. Deformation statistics are therefore radically different from those of turbulence, and since breakup occurs whenever the bubble elongation is larger than a certain threshold, the breakup probabilities also strongly differ from those of turbulence.

The present work reports an experimental investigation of an air bubble immersed in a uniform downward turbulent flow of water, which has been characterized by means of fast PIV. Thanks to rapid imaging of three perpendicular views combined with three-dimensional shape recognition, temporal evolutions of bubble translation, rotation and deformation have been measured.

The dynamics of the bubble involves three mechanisms fairly decoupled:

- The average shape of the bubble is imposed by the sliding between the phases. It is approximately a oblate spheroid with its small axis in the direction of motion. It therefore mainly involves spherical harmonic $n=2, m=0$.

- The wake instabilities lead to quasi-periodic oscillations of velocity and orientation but only cause slight shape oscillations, which are not axisymmetric in agreement with previous works dealing with bubbles rising in a quiescent fluid. ${ }^{10,11}$ These deformations do not play a significant role in the breakup.

- Turbulence generates random deformations that may cause breakup when the bubble elongation exceeds twice the equivalent diameter, a value in agreement with results obtained in the absence of buoyancy.

A large deformation occurs when the bubble encounters an intense turbulent eddy. It corresponds to an elongation in a horizontal direction, which breaks the axial symmetry of the average shape and mainly involves spherical harmonic $n=2, m=2$. After interaction with the eddy, the bubble either breaks or relaxes towards its average shape on a time scale close to $f_{2}^{-1}$, without experiencing any oscillations. The existence of a large sliding motion between the phases thus radically changes the shape dynamics: it could still be modeled by an oscillator - which describes now the amplitude of mode $n=2, m=2-$ with a proper frequency $f_{2}$ but with 
a damping rate comparable to $f_{2}$. With such an overdamped oscillator, breakup cannot result from stochastic resonance and is always caused by the interaction with a single intense eddy.

This absence of memory of the shape dynamics has a major consequence for breakup. The statistics of waiting times between two large deformations and, similarly, the statistics of bubble residence time before breakup, do not involve the characteristic timescale $f_{2}^{-1}$ of the bubble deformation and are identical to the statistics of turbulence. The response time $f_{2}^{-1}$ however controls the effectiveness of turbulent bursts - through the Weber number - and the duration of the breakup phenomenon.

Unexpectedly, breakup statistics in a turbulent flow eventually turn out to be easier to model for a large bubble rising on an oscillatory path than for a non-sliding bubble in microgravity condition. It is due to the strong enhancement of the damping of shape oscillations. The reason of this enhancement and its relation with the existence of a significant sliding remains an open question that deserves to be addressed in a future work.

${ }^{1}$ F. Risso and J. Fabre. Oscillations and breakup of a bubble immersed in a turbulent field. J. Fluid Mech., 372:323, 1998.

${ }^{2}$ S. Galinat, F. Risso, O. Masbernat, and P. Guiraud. Dynamics of drop breakup in inhomogeneous turbulence at various volume fractions. J. Fluid Mech., 578:85, 2007.

${ }^{3} \mathrm{~F}$. Risso. The mechanisms of deformation and breakup of drops and bubbles. Multiphase Sci. Tech., 12:1, 2000.

${ }^{4}$ C. A. Miller and L. E. Scriven. The oscillations of a fluid droplet immersed in another fluid. J. Fluid Mech., 32:417-435, 1968.
${ }^{5}$ A. Prosperetti. Free oscillations of drops and bubbles: The initial-value problem. J. Fluid Mech., 100:333-347, 1980.

${ }^{6} \mathrm{~S}$. V. Subramanyam. A note on the damping and oscillations of a fluid drop moving in another fluid. J. Fluid Mech., 37:715-725, 1969

${ }^{7}$ O. A. Basaran, T. C. Scott, and C. H. Bayers. Drop oscillations in liquid-liquid systems. AIChE J., 440:1263, 1989.

${ }^{8}$ D. I. Meiron. On the stability of gas bubbles rising in an inviscid fluid. J. Fluid Mech., 198:101-114, 1989.

${ }^{9} \mathrm{~K}$. Ellingsen and F. Risso. On the rise of an ellipsoidal bubble in water: oscillatory paths and liquid-induced velocity. J. Fluid Mech., 440:235, 2001.

${ }^{10} \mathrm{~K}$. Lunde and R. J. Perkins. Shape oscillations of rising bubbles. Applied Scientific Research, 58:387-408, 1997.

${ }^{11}$ C. Veldhuis, A. Biesheuvel, and L. van Wijngaarden. Shape oscillations on bubbles rising in clean and in tap water. Phys. Fluids, 20:040705, 2008.

${ }^{12} \mathrm{~K}$. Wichterle, J. Wichterlová, and L. Kulhánková. Breakup of bubbles rising in liquids of low and moderate viscosity. Chemical Engineering Communications, 192:550-556, 2005.

${ }^{13}$ S. M. Smith and J. M. Brady. SUSAN - a new approach to low level image processing. Int. Journal of Computer Vision, 23:45, 1997.

${ }^{14} \mathrm{~L}$. Ji and H. Yan. Attractable snakes based on the greedy algorithm for contour extraction. Pattern Recognition, 35:791, 2002.

${ }^{15}$ T. Kayikcioglu, A. Gangal, and M. Ozer. Reconstructing ellipsoids from three projection contours. Pattern Recognition Letters, 21:959, 2000.

${ }^{16} \mathrm{~J}$. Westerweel and F. Scarano. Universal outlier detection for PIV data. Experiments in Fluids, 39(6):1096, 2005.

${ }^{17}$ J. T. Lindt. On the periodic nature of the drag on a rising bubble. Chem. Eng. Sci., 27:1775-1781, 1972. 